\title{
ON AXIOMATIC CHARACTERISATION OF GENERALIZED QUALITATIVE MEASURE OF VARIATION OF INFORMATION THROUGH GENERATING FUNCTION
}

\author{
R.P. SINGH AND J.D. BHARDWAJ
}

\begin{abstract}
The aim of the present contribution is to define a generalized qualitative measure of variation of information through generating function and to characterize it axiomatically. In this sequence the generalized qualitative measure of information improvement as the difference of generalized measure of information of variation defined earlier is also defined. Most of the measures studied so far thus turns out to be particular ones. Some basic applicative properties are discussed.
\end{abstract}

\section{Introduction}

The study of directed divergence has shown remarkable impact in many fields such as national income, inequality, racial segregation, political alignment, combination of statements in balance sheets, questionnaire theory, decision theory and so on.

The impact of "qualitative one" was effectively introduced by Belis and Guiasu [2] who in turn extended the Shannon's entropy to weighted entropy. Later Taneja, [9] introduced the idea of weighted (qualitative) directed divergence. The authors [14] defined qualitative measure of variation of information of type $\beta, \beta>1$ viz.

$$
I_{n}^{\beta}\left(P ; U ; W_{p}\right)=\sum_{i=1}^{n} w_{p_{i}}^{\beta} p_{i}^{\beta} \log \left(\frac{p_{i}}{u_{i}}\right) \quad \beta \neq 0 ; \beta>0
$$

for the weighted information scheme:

$$
S=\left(\begin{array}{ccc}
E_{1} & \cdots & E_{n} \\
p_{1} & \cdots & p_{n} \\
u_{1} & \cdots & u_{n} \\
w_{p_{1}} & \cdots & w_{p_{n}}
\end{array}\right)=\left(\begin{array}{c}
E \\
p \\
U \\
W_{p}
\end{array}\right)
$$

where $E_{1}, \ldots, E_{n}$ are the partition events of $E$ whose corresponding probability distribution $P=\left(p_{1}, \ldots, p_{n}\right)$ and the revised probability distribution, corresponding to $P$ is $U=\left(u_{1}, \cdots, u_{n}\right)$ where qualitative distribution is $W_{p}=\left(w_{p_{1}}, \ldots, w_{p_{n}}\right)$ corresponds to $P=\left(p_{1}, \cdots, p_{n}\right)$.

Received August 16, 1989. 
(1.1) includes the weighted divergence studied by Taneja [9] as a particular case when $\beta=1$ and Kullback-Leibler divergence [7] when $\beta=1, w_{p_{i}}=1 \forall i=1,2, \cdots, n$.

We all are aware of the fact that the generating function's successive derivatives at the point 0 give the successive moments of a probability distribution, if these moments exist. This technique to define new qualitative measure of variation of information of type $\beta, \beta>1, \beta \neq 0$ has been presented by the authors in the earlier paper [14]. In this communication our aim is to develop this technique to define generalized qualitative measure of variation of information as it is more effective and more flexible from the applicative point of view. This has been done in the next section while section 3 deals with the generalized information improvment. Section 4 investigates the axiomatic characterization of the generalized qualitative measure of variation of information. While the last section presents the basic applicative properties of generalized qualitative information improvement.

\section{Generalized qualitative measure of variation of information through gen- erating function}

Let $(X ; W ; m)$ be the measure space. The initial measure $m$ is the Leibesgue measure in the continuous case and the measure assigning the unit to each point in the discrete case. Let $\nu$ be the reference measure, and $\mu, \eta$ the probability and qualitive measure respectively. On the same space $X$ such that $\mu \leq \eta \leq m$ where " $<$ " measure "absolutely continuous with respect to".

Let

$$
f=\frac{d \mu}{d m} ; \quad g=\frac{d \nu}{d m} ; \quad h=\frac{d \eta}{d m}
$$

be the corresponding densities (The Radon-Nikodym derivatives). Let $g$ and $h$ be strictly positive, $m$ almost everywhere (c.f. Guiasu [4] 1985). The generalized Relative Parametric Qualitative Information Generating Function is defined:

$$
\begin{aligned}
R_{a}^{b, c}(f ; g ; h ; t)= & \frac{(t-1)}{\left[2^{(a-1) b c}-1\right]}\left[\left\{\sum_{i=1}^{n} h^{b}\left(w_{n}\right) \cdot f^{a b}(n) g^{(1-a) c}(n)\right\}^{c}\right. \\
& \left.-\left\{\sum_{i=1}^{n} h\left(w_{h}\right)^{b} f^{b}(n)\right\}^{c}\right]^{(2 t-1)}
\end{aligned}
$$

where $a, b, c$ are arbitrary parameters, $t>0, t \neq 1$. Diff. (2.1) w.r.t. " $t$ ", we get

$$
\begin{aligned}
& R_{c}^{\prime(b, c)}(f ; g ; h ; t) \\
= & \frac{1}{\left[2^{(a-1) b c}-1\right]}\left[\left\{\sum_{i=1}^{n} h^{b}\left(w_{n}\right) f^{a b}(n) g^{(1-a) b}(n)\right\}^{c}-\left\{\sum_{i=1}^{n} h^{b}\left(w_{n}\right) f^{b}(n)\right\}^{c}\right]^{(2 t-1)} \\
& +\frac{(t-1)}{\left[2^{(a-1) b c}-1\right]}\left[\left\{\sum_{i=1}^{n} h^{b}\left(w_{n}\right) f^{a b}(n) g^{(1-a) b}(n)\right\}^{c}-\left\{\sum_{i=1}^{n} h^{b}\left(w_{n}\right) f^{b}(n)\right\}^{c}\right]^{(2 t-1)}
\end{aligned}
$$




$$
\cdot \log \left[\left\{\sum_{i=1}^{n} h^{b}\left(w_{n}\right) f^{a b}(n) g^{(1-a) b}(n)\right\}^{2}-\left\{\sum_{i=1}^{n} h^{b}\left(w_{n}\right) f^{b}(n)\right\}^{c}\right]
$$

Put $t=1$ in (2.2), we have

$$
\begin{aligned}
R_{a}^{\prime(b, c)}(f ; g ; h ; 1)= & \frac{1}{\left[2^{(a-1) b c}-1\right]}\left[\left\{\sum_{i=1}^{n} h^{b}\left(w_{n}\right) f^{a b}(n) g^{(1-a) b}(n)\right\}^{c}\right. \\
& \left.-\left\{\sum_{i=1}^{n} h^{b}\left(w_{n}\right) f^{b}(n)\right\}\right]
\end{aligned}
$$

(2.3) is interpreted as the generating function of the generalized qualitative measure of variation of information. Now setting $f(n)=p_{i} ; g(n)=u_{i} ; h\left(w_{n}\right)=w_{p_{i}}$, then (2.3)
becomes

$$
I_{a}^{(b, c)}\left(P ; U ; W_{p}\right)=\frac{1}{\left[2^{(a-1) b c}-1\right]}\left\{\left[\sum_{i=1}^{n} w_{p_{i}}^{b} p_{i}^{a b} u_{i}^{(1-a) b}\right]^{c}-\left[\sum_{i=1}^{n} w_{p_{i}}^{b} p_{i}^{b}\right]^{c}\right\}
$$

This (2.4) is interpreted as the generalized qualitative measure of variation of information (or weighted divergence of order a and type $(b, c)$ ).

(2.4) reduces to

$$
I_{a}^{(b, c)}(P ; U)=\frac{1}{\left[2^{(a-1) b c}-1\right]}\left[\left\{\sum_{i=1}^{n} p_{i}^{a b} u_{i}^{(1-a) b}\right\}^{c}-\left\{\sum_{i=1}^{n} p_{i}^{b}\right\}^{c}\right]
$$

the generalized measure of variation of information (or divergence of order a and type $(b, c))$ by setting $w_{p_{i}}=1 \forall i=1, \cdots, n$.

\section{Particular and limiting cases of (2.4) and (2.5)}

(a) when $c=1$

(i) (2.4) becomes

$$
I_{a}^{(b, 1)}\left(P ; U ; W_{p}\right)=\frac{1}{\left[2^{(a-1) b}-1\right]}\left[\left\{\sum_{i=1}^{n} w_{p_{i}}^{b} p_{i}^{a b} u_{i}^{(1-a) b}\right\}-\left\{\sum w_{p_{i}}^{b} p_{i}^{b}\right\}\right]
$$

which is interpreted as weighted divergence of order $a$ and type $b$.

(ii) (2.5) becomes

$$
I_{a}^{(b, 1)}(P ; U)=\frac{1}{\left[2^{(a-1) b}-1\right]}\left[\left\{\sum_{i=1}^{n} p_{i}^{a b} u_{i}^{(1-a) b}\right\}-\left\{\sum_{i=1}^{n} p_{i}^{b}\right\}\right]
$$

which is called as divergence of order $a$ and type $b$.

(b) when $a \rightarrow 1$ 
(i) (2.6) becomes

$$
I_{1}^{(b, 1)}\left(P ; U ; W_{p}\right)=\sum_{i=1}^{n} w_{p_{i}}^{b} p_{i}^{b} \log \frac{p_{i}}{u_{i}}
$$

which is (Singh and Bhardwaj [14]) weighted divergence of type $b$ and (2.7) gives

(ii)

$$
I_{1}^{(b, 1)}(P ; U)=\sum_{i=1}^{n} p_{i}^{b} \log \frac{p_{i}}{u_{i}}
$$

(c) when $b=1$

which is (Kapur's [6] divergence of type $b$.

(i) (2.8) becomes

$$
I_{1}^{(1,1)}\left(P ; U ; W_{p}\right)=\sum_{i=1}^{n} w_{p_{i}} p_{i} \log \frac{p_{i}}{u_{i}}
$$

(Taneja's [9] weighted divergence.)

(ii) (2.9) becomes

$$
I_{1}^{(1,1)}(P ; U)=\sum_{i=1}^{n} p_{i} \log \frac{p_{i}}{u_{i}}
$$

(Kullback-Leibler's divergence [7]), also (2.11) is obtained through (2.10) when $w_{p_{i}}=1 \forall i=1,2, \cdots, n$.

\section{Generalized qualitative information improvement}

It is a well known fact that the information improvement generating function is obtained as the difference of generating functions of measures of variations of information. Therefore we have

$$
\begin{aligned}
R_{a}^{\prime(b, 1)}\left(f ; g_{1} ; h, 1\right)= & {\left[2^{(a-1) b c}-1\right]^{-1}\left[\left\{\sum_{i=1}^{n} h^{b}\left(w_{n}\right) f^{a b}(n) g_{1}^{(1-a) b}\right\}^{c}\right.} \\
& \left.-\left\{\sum_{i=1}^{n} h^{b}\left(w_{n}\right) f^{b}(n)\right\}^{c}\right] \\
R_{a}^{\prime(b, 1)}\left(f ; g_{2} ; h, 1\right)= & {\left[2^{(a-1) b c}-1\right]^{-1}\left[\left\{\sum_{i=1}^{n} h^{b}\left(w_{n}\right) f^{a b}(n) g_{2}^{(1-a) b}\right\}^{c}\right.} \\
& \left.-\left\{\sum_{i=1}^{n} h^{b}\left(w_{n}\right) f^{b}(n)\right\}^{c}\right]
\end{aligned}
$$

Hence the generalized qualitative generating function of information improvement of order $a$ and type $(b, c)$ is given by :

$$
R_{a}^{(b, c)}\left(f ; g_{1} ; h ; 1\right)-R_{a}^{\prime(b, c)}\left(f ; g_{2} ; h ; 1\right)
$$




$$
=\left[2^{(a-1) b c}-1\right]^{-1}\left[\left\{\sum_{i=1}^{n} h^{b}\left(w_{n}\right) f^{a b}(n) g_{1}^{(1-a) b}\right\}^{c}-\left\{\sum_{i=1}^{n} h^{b}\left(w_{n}\right) f^{a b}(n) g_{2}^{(1-a) b}\right\}^{c}\right]
$$

Setting, $h\left(w_{n}\right)=w_{p_{i}} ; f(n)=p_{i} ; g_{1}(n)=u_{i} ; g_{2}(n)=r_{i}$ in (3.3) we get

$$
\begin{aligned}
I_{a}^{(b, c)}\left(P ; U ; R ; W_{p}\right)= & {\left[2^{(a-1) b c}-1\right]^{-1}\left[\left\{\sum_{i=1}^{n} w_{p_{i}}^{b} p_{i}^{a b} u_{i}^{(1-a) b}\right\}^{c}\right.} \\
& \left.-\left\{\sum_{i=1}^{n} w_{p_{i}}^{b} p_{i}^{a b} r_{i}^{(1-a) b}\right\}^{c}\right]
\end{aligned}
$$

This is interpreted as the generalized qualitative information improvement (or qualitative information improvement of order $a$ and type $(b, c))$.

Setting $w_{p_{i}}=1 \forall i=1,2, \cdots, n,(3.4)$ gives

$$
I_{a}^{(b, c)}(P ; U ; R)=\left[2^{(a-1) b c}-1\right]^{-1}\left[\left\{\sum_{i=1}^{n} p_{i}^{a b} u_{i}^{(1-a) b}\right\}^{c}-\left\{\sum_{i=1}^{n} p_{i}^{a b} r_{i}^{(1-a) b}\right\}^{c}\right]
$$

which is Information Improvement of order $a$ type $(b, c)$.

Particular and limiting cases of (3.4) and (3.5)

(a) when $c=1$

(i) (3.4) becomes

$$
\begin{aligned}
I_{a}^{(b, 1)}\left(P ; U ; R ; W_{p}\right)= & {\left[2^{(a-1) b}-1\right]^{-1}\left[\left\{\sum_{i=1}^{n} w_{p_{i}}^{b} p_{i}^{a b} u_{i}^{(1-a) b}\right\}\right.} \\
& \left.-\left\{\sum_{i=1}^{n} w_{p_{i}}^{b} p_{i}^{a b} r_{i}^{(1-a) b}\right\}\right]
\end{aligned}
$$

This can be interpreted as W.I.I. of order $a$ and type $b$.

(ii) Now (3.5) gives

$$
\begin{aligned}
I_{a}^{(b, c)}(P ; U ; R) & =I_{a}^{(b, 1)}(P ; U ; R) \\
& =\left[2^{(a-1) b}-1\right]^{-1}\left[\left\{\sum_{i=1}^{n} p_{i}^{a b} u_{i}^{(1-a) b}\right\}-\left\{\sum_{i=1}^{n} p_{i}^{a b} r_{i}^{(1-a) b}\right\}\right]
\end{aligned}
$$

(b) $a \rightarrow 1$

which is termed as the I.I. of order $a$ and type $b$.

(i) (3.6) becomes

$$
I_{1}^{(b, 1)}\left(P ; U ; R ; W_{p}\right)=\sum_{i=1}^{n} w_{p_{i}}^{b} p_{i}^{b} \log \left(\frac{r_{i}}{u_{i}}\right)
$$


Singh and Bhardwaj [15] W.I.I. of type $b$.

(ii) (3.7) becomes

$$
I_{a}^{(b, 1)}(P ; U ; R)=I_{1}^{(b, 1)}(P ; U ; R)=\sum_{i=1}^{n} p_{i}^{b} \log \frac{r_{i}}{u_{i}}
$$

Kapur's I.I. of type b. [5].

(c) when $b=1$

(i) (3.8) takes the form

$$
I_{1}^{(1,1)}\left(P ; U ; R ; W_{p}\right)=\sum_{i=1}^{n} w_{p_{i}} p_{i} \log \frac{r_{i}}{u_{i}}
$$

Singh and Bhardwaj [13] W.I.I.

(ii) (3.9) becomes

$$
I_{1}^{(b, 1)}(P ; U ; R)=\sum_{i=1}^{n} p_{i} \log \left(\frac{r_{i}}{u_{i}}\right)
$$

Theil's measure [16].

(d) when $b=1$

(i) (3.6) becomes

$$
I_{a}^{(1,1)}\left(P ; U ; R ; W_{p}\right)=\left[2^{(a-1)}-1\right]^{-1}\left\{\left[\sum_{i=1}^{n} w_{p_{i}} p_{i}^{a} u_{i}^{1-a}\right]-\left[\sum_{i=1}^{n} w_{p_{i}} p_{i}^{a} r_{i}^{1-a}\right]\right\}
$$

W.I.I. of order $a$.

(ii) (3.7) becomes

$$
I_{a}^{(1,1)}(P ; U ; R)=\left[2^{(a-1)}-1\right]^{-1}\left[\sum_{i=1}^{n} p_{i}^{a} u_{i}^{1-a}-\sum_{i=1}^{n} p_{i}^{a} r_{i}^{1-a}\right]
$$

I.I. of order a defined by Kapur [5].

4. Axiomatic characterization of generalized qualitative measure of variation of information

Theorem 4.1. Let $F:[0,1] \times[0,1] \times[0, \infty] \rightarrow R$ (reals) be a function satisfying the following axioms:

(i)

$$
F\left(P ; U ; W_{p}\right)=K_{1}\left[\sum_{i=1}^{n} f\left(p_{i}, u_{i}, w_{p_{i}}\right)\right]^{c}+K_{2} \bar{W}_{p}^{c}
$$


where $K_{1}, K_{2}, c \neq 0$, where $f$ is a real valued continuous function defined over $[0,1] \times$ $[0,1] \times[0, \infty] . K_{1}, K_{2}$ are arbitrary constants and $c$ is an arbitrary parameter.

(ii)

$$
\begin{aligned}
F\left(P^{*} Q ; U^{*} V ; W_{p}^{*} W_{Q}\right)= & \bar{W}_{Q}^{c} F\left(P ; U ; W_{p}\right)+\bar{W}_{p}^{c} F\left(Q, V ; W_{Q}\right) \\
& -\frac{1}{K_{2}} F\left(P ; U ; W_{p}\right)-F\left(Q, V, W_{Q}\right)
\end{aligned}
$$

where

$$
\begin{aligned}
& P^{*} Q=p_{i} q_{i} \quad \forall p_{i} \in P \text { and } q_{j} \in Q \\
& U^{*} V=u_{i} v_{i} \quad \forall u_{i} \in U \text { and } v_{j} \in V \\
& W_{p}^{*} W_{Q}=w_{p_{i}} w_{q_{i}} \forall w_{p_{i}} \in W_{p} \text { and } w_{q_{i}} \in W_{Q} \forall\left\{\begin{array}{l}
i=1,2, \cdots, n \\
j=1,2, \cdots, m
\end{array}\right. \\
& \bar{W}_{p}=\sum_{i=1}^{n} w_{p_{i}}^{b} p_{i}^{b} \text { and } \bar{W}_{Q}=\sum_{i=1}^{m} w_{q_{i}}^{b} q_{j}^{b}
\end{aligned}
$$

(iii)

then

$$
F\left(1,0 ; \frac{1}{2}, \frac{1}{2} ; 1 ; 0\right)=1
$$

$$
F\left(P ; U ; W_{p}\right)=I_{a}^{(b, c)}\left(P ; U ; W_{p}\right)
$$

(All logarithms are taken as base 2).

Proof. Considering axiom (ii)

$$
\begin{aligned}
F\left(P^{*} Q ; U^{*} V ; W_{p}^{*} W_{Q}\right)= & \bar{W}_{Q}^{c} F\left(P ; U ; W_{p}\right)+\bar{W}_{p}^{c} F\left(Q ; V ; W_{Q}\right) \\
& -\frac{1}{K_{2}} F\left(P ; U ; W_{p}\right) F\left(Q ; V ; W_{Q}\right)
\end{aligned}
$$

Using axiom (i) in the above, we have

$$
\begin{aligned}
& K_{1}\left[\sum_{i=1}^{n} \sum_{j=1}^{m} f\left(p_{i} q_{j} ; u_{i} v_{j} ; w_{p_{i}} w_{q_{j}}\right)\right]^{c}+K_{2}\left[\sum_{i=1}^{n} \sum_{j=1}^{m} w_{p_{i}}^{b} p_{i}^{b} w_{q_{j}}^{b} q_{j}^{b}\right]^{c} \\
= & {\left[\sum_{j=1}^{n} w_{q_{j}}^{b} q_{j}^{b}\right]^{c}\left[K_{1}\left\{\sum_{i=1}^{n} f\left(p_{i}, u_{i}, w_{p_{i}}\right)\right\}^{c}+K_{2}\left\{\sum w_{p_{i}}^{b} p_{i}^{b}\right\}^{c}\right] } \\
& +\left[\sum_{i=1}^{n} w_{p_{i}}^{b} p_{i}^{b}\right]^{c}\left[K_{1}\left\{\sum_{i=1}^{n} f\left(q_{j} ; v_{j} ; w_{q_{i}}\right)\right\}^{c}\right. \\
& \left.+K_{2}\left\{\sum_{i=1}^{m} w_{q_{j}}^{b} q_{j}^{b}\right\}^{c}\right]-\frac{1}{K_{2}}\left[K _ { 1 } \left\{\sum_{i=1}^{n} f\left(p_{i} ; u_{i} ; w_{p_{i}}\right\}^{c}\right.\right. \\
& \left.+K_{2}\left\{\sum_{i=1}^{n} w_{p_{i}}^{b} p_{i}^{b}\right\}^{c}\right]\left[K_{1}\left\{\sum_{i=1}^{m} f\left(q_{j} ; v_{j} ; w_{q_{j}}\right)\right\}^{c}+K_{2}\left\{\sum_{i=1}^{m} w_{q_{j}}^{b} q_{j}^{b}\right\}^{c}\right]
\end{aligned}
$$


Dividing both sides by

$$
\bar{W}_{p}^{c} \bar{W}_{Q}^{c}=\left[\sum_{i=1}^{n} w_{p_{i}}^{b} p_{i}^{b}\right]^{c}\left[\sum_{i=1}^{m} w_{q_{j}}^{b} q_{j}^{b}\right]^{c}=\left[\sum_{i=1}^{n} \sum_{j=1}^{m} w_{p_{i}}^{b} p_{i}^{b} w_{q_{j}}^{b} q_{j}^{b}\right]^{c}
$$

and substituting

$$
\sum_{i=1}^{n} f_{1}\left(p_{i}, u_{i}, w_{p_{i}}\right)=\frac{\sum_{i=1}^{n} f\left(p_{i}, u_{i}, w_{p_{i}}\right)}{\sum_{i=1}^{n} w_{p_{i}}^{b} p_{i}^{b}} \quad \text { in (4.5) }
$$

We get

$$
\left[\sum_{i=1}^{n} \sum_{j=1}^{m} f_{1}\left(p_{i} q_{j} ; u_{i} v_{j} ; w_{p_{i}} w_{q_{j}}\right)\right]^{c}=\left(-\frac{K_{1}}{K_{2}}\right)\left[\sum_{i=1}^{n} f_{1}\left(p_{i}, u_{i}, w_{p_{i}}\right)\right]^{c}\left[\sum_{j=1}^{m} f_{1}\left(q_{j}, v_{j}, w_{q_{j}}\right)\right]^{c}
$$

or

$$
\sum_{i=1}^{n} \sum_{j=1}^{m} f_{1}\left(p_{i} q_{j} ; u_{i} v_{j} ; w_{p_{i}} w_{q_{j}}\right)=\left(-\frac{K_{1}}{K_{2}}\right)^{1 / c}\left[\sum_{i=1}^{n} \sum_{j=1}^{m} f_{1}\left(p_{i}, u_{i}, w_{p_{i}}\right) f_{1}\left(q_{j}, v_{j}, w_{q_{j}}\right)\right]
$$

Equivalently, we can consider (4.7) as

$$
f_{1}\left(p q ; u v ; w_{p} w_{q}\right)=\left(-\frac{K_{1}}{K_{2}}\right)^{1 / c}\left[f_{1}\left(p ; u ; w_{p}\right) f_{1}\left(q ; v ; w_{q}\right)\right]
$$

for all reals, $p, q, u, v \in[0,1] ; w_{p}, w_{q} \in[0, \infty]$ (i.e. $w_{p}, w_{q}>0$ ). Substituting

$$
\left(-\frac{K_{1}}{K_{2}}\right)^{1 / c} f_{1}\left(p ; u ; w_{p}\right)=\phi\left(p, u, w_{p}\right)
$$

(4.8) becomes

$$
\phi\left(p q, u v, w_{p} w_{q}\right)=\phi\left(p, u ; w_{p}\right) \phi\left(q, v ; w_{q}\right)
$$

The most general continuous solution of the functional equation (4.10) [refer Aczel [1]] is given by

$$
\phi\left(p ; u, w_{p}\right)=p^{\alpha} U^{\beta} w_{p}^{\gamma} \quad \text { where } \alpha>0, \beta \neq 0
$$

Write

$$
\alpha=(a-1) b, \quad \beta=(1-a) b ; \quad \gamma=0
$$

Then

$$
\phi\left(p ; u ; w_{p}\right)=p^{(a-1)^{b}} u_{i}^{(1-a)^{b}}
$$

from (4.11), (4.9) and (4.1) we have

$$
F\left(P ; U, W_{p}\right)=\left(-k_{2}\right)\left\{\left[\sum_{i=1}^{n} p_{i}^{a b} u_{i}^{(1-a)^{b}} w_{p_{i}}^{b}\right]^{c}-\left[\sum_{i=1}^{n} w_{p_{i}}^{b} p_{i}^{b}\right]^{c}\right\}
$$


using (4.3) and (4.12) we get

$$
-k_{2}=\frac{1}{\left[2^{(a-1)^{b c}}-1\right]}
$$

Hence (4.12) takes the form

$$
I_{a^{b}}^{b, c}\left(P ; U ; W_{p}\right)=\frac{1}{\left[2^{(a-1)^{b c}}-1\right]}\left\{\left[\sum_{i=1}^{n} w_{p_{u}}^{b} p_{i}^{a b} u_{i}^{(1-a) b}\right]^{c}-\left[\sum w_{p_{i}}^{b} p_{i}^{b}\right]^{c}\right\}
$$

This completes the proof.

5. Properties of information improvement. $I_{a}^{(b, c)}\left(P ; U, R ; W_{p}\right)$

The beauty of any good measure lies in its properties as emphasised by Kapur (5). According to him, in most of the measures as we go from the true distribution $P$ to any other distribution $U$, there is a positive information improvement, which is a highly undesirable property.

Secondly, the generalised measure of information improvement should approach the useful known Theil's measure; is not enough. We would like to have a measure which should have as many as posible, idealy all, properties of Theil's measure. Here the measure $I_{a}^{(b, c)}\left(P ; U ; R ; W_{P}\right)$ i.e. (3.4) satisfies most of the properties discussed in this section.

The generalized measure of weighted information improvement of order $a$ and type $(b, c)$ is defined as

$$
D_{a}^{(b, c)}\left(P ; U ; W_{p}\right)-D_{a}^{(b, c)}\left(P ; R ; W_{p}\right)
$$

whereas, Theil measure is

$$
I(P ; U ; R)=D(P, U)-D(P, R)
$$

and the generalized (weighted) information improvement i.e. (3.4) as :

$$
I_{a}^{(b, c)}\left(P ; U ; R ; W_{p}\right)=D_{a}^{(b, c)}\left(P ; U ; W_{p}\right)-D_{a}^{(b, c)}\left(P ; R ; W_{p}\right)
$$

The following properties can be easily verified:

(i) If we go from distribution $U$ to the required (True) distribution $P$, there is always a positive qualitative (weighted) information improvement and will be zero iff $P=U$.

(a) $I(P ; U ; P)=D(P, U)-D(P, P)$ for Theil's measure

$$
=D(P, U) \geq 0
$$

(b) $I_{a}^{(b, c)}\left(P ; U ; P ; W_{p}\right)=D_{a}^{(b, c)}\left(P, U, W_{p}\right)-D_{a}^{(b, c)}\left(P ; P ; W_{p}\right)$

$$
=D_{a}^{(b, c)}\left(P ; U ; W_{p}\right) \geq 0 \text {. }
$$


(ii) If $P \rightarrow P$

$$
\begin{aligned}
& U \rightarrow P \\
& R \rightarrow U
\end{aligned}
$$

i.e. if we go from true distribution $P$ to any other distribution, then the information improvement is negative unless we go from $P$ to $P$ itself when the information improvement is zero.

$$
\begin{aligned}
& \text { (a) } \begin{aligned}
I(P ; P ; U) & =D(P, P)-D(P, U) \\
& =-D(P, U) \leq 0 \quad \text { for Theil's measure }
\end{aligned} \\
& \text { (b) } \begin{aligned}
I_{a}^{(b, c)}\left(P ; P ; U ; W_{p}\right) & =D_{a}^{(b, c)}\left(P, P, W_{p}\right)-D_{a}^{(b, c)}\left(P ; U ; W_{p}\right) \\
& =-D_{a}^{(b, c)}\left(P ; U ; W_{p}\right) \leq 0 .
\end{aligned}
\end{aligned}
$$

(iii) The total information improvement in going from $U$ to $T$ is the same as going from $U$ to $R$, then from $R$ to $S$ and finally from $S$ to $T$, i.e. Total information improvement depends only on the initial and final distribution and not on the intermediate distribution.

(a) $I(P ; U ; R)+I(P ; R ; S)+I(P ; S ; T)=I(P, U, T)$ for Theil's measure

(b) $\quad I_{a}^{(b, c)}\left(P ; U ; R, W_{p}\right)+I_{a}^{(b, c)}\left(P ; R ; S ; W_{p}\right)+I_{a}^{(b, c)}\left(P, S, T, W_{p}\right)$

$$
\begin{aligned}
= & {\left[D_{a}^{(b, c)}\left(P ; U ; W_{p}\right)-D_{a}^{(b, c)}\left(P, R, W_{p}\right)\right]+\left[D_{a}^{(b, c)}\left(P, R, W_{p}\right)-D_{a}^{(b, c)}\left(P, S, W_{p}\right)\right] } \\
& +\left[D_{a}^{(b, c)}\left(P ; S ; W_{p}\right)-D_{a}^{(b, c)}\left(P ; T ; W_{p}\right)\right] \\
= & {\left[D_{a}^{(b, c)}\left(P, U, W_{p}\right)-D_{a}^{(b, c)}\left(P ; T ; W_{p}\right)=I_{a}^{(b, c)}\left(P ; U ; T ; W_{p}\right) .\right.}
\end{aligned}
$$

(iv) (a) $I(P, U, R)$ is a convex function of $U$ and a concave function of $R$.

(b)

$$
\begin{aligned}
& \frac{d^{2} I_{a}^{(b, c)}\left(P, U, R, W_{p}\right)}{d U^{2}} \\
= & \frac{1}{\left(2^{(a-1) b c}\right)}\left\{c \left\{(c-1)\left[\sum_{i=1}^{n} w_{p_{i}}^{b} p_{i}^{a b} u_{i}^{(1-a) b}\right]^{(c-2)}\right.\right. \\
& {\left.\left[\sum_{i=1}^{n} w_{p_{i}}^{b} p_{i}^{a b}(a-1) b u_{i}^{(a-1) b-1}\right]\left[\sum_{i=1}^{n} w_{p_{i}}^{b} p_{i}^{a b}(a-1) b u_{i}^{(a-1) b-1}\right]\right\} } \\
+ & \sum_{i=1}^{n}\left[w_{p_{i}}^{b} p_{i}^{a b} u_{i}^{(a-1) b}\right]^{c-1}\left\{\sum_{i=1}^{n} w_{p_{i}}^{b} p_{i}^{a b}(a-1)[(a-1) b-1] u_{i}^{(a-1) b-2}\right\}>0
\end{aligned}
$$

convex function for $u$ wnen $a>1, b>1, c>1$. 
Similarly

$$
\begin{aligned}
& \frac{d^{2} I_{a}^{(b, c)}\left(P ; U ; R ; W_{p}\right)}{d R^{2}} \\
= & \frac{(-1)}{\left(2^{(a-1) b c}\right)}\left\{c(c-1)\left[\sum_{i=1}^{n} w_{p_{i}}^{b} p_{i}^{a b} r_{i}^{(a-1) b}\right]^{(c-2)}\right. \\
& {\left[\sum_{i=1}^{n} w_{p_{i}}^{b} p_{i}^{a b}(a-1) b r_{i}^{(a-1) b-1}\right]\left[\sum_{i=1}^{n} w_{p_{i}}^{b} p_{i}^{a b}(a-1) b r_{i}^{(a-1) b-1}\right] } \\
+ & {\left.\left[\sum_{i=1}^{n} w_{p_{i}}^{b} p_{i}^{a b} r_{i}^{(a-1) b-1}\right]^{c-1}\left[\sum_{i=1}^{n} w_{p_{i}}^{b} p_{i}^{a b}((a-1)(b)-1)(a-1) b r_{i}^{(a-1) b-2}\right]\right\} \leq 0 }
\end{aligned}
$$

so a concave function of $R$ when $a>1, b>1, c>1$.

Also this (3.4) measure reduces to most of the known measures including Theil's measure as shown in section 3. Hence the characterization of this measure (3.4) will strengthen its applicability more as the basic properties are satisfied. Its characterization will be done elsewhere.

\section{References}

[1] Aczel, J., "Lectures on Functional Equations and their Applications" Academic Press, New York (1966).

[2] Belis and Guiasu, "A quantitative- qualitative measure of information in cybernatics systems", IEEE Trans. Inform. Theory, $14: 593-594$ (1968).

[3] Golombs, S., "The information generating function of a probability distribution", IEEE Trans. Theory. IT-12, 75-77 (1966).

[4] Guiasu, S., "The Relative Information generating function", Information Sciences, 35: 235-241, (1985).

[5] Kapur, J.N., "New Measures of Information Improvement", JCISS Vol.11 (1) 39-46 (1986).

[6] Kapur, J.N., "On Entropy and directed divergence of order $\alpha$ and type $\beta$ " Advances in Management Studies, Vol.3 (3-4) 129-144.

[7] Kullback-Leibler, "On Information and Sufficiency" Ann. Math. Statist. 22, 79-86 (1951).

[8] Longo G., "Quantitative Qualitative Measure of Information" Springer, New York (1972).

[9] Taneja, H.C., "On the quantitative-qualitative measure of relative information", Information Sciences, 33 : 223-227 (1984).

[10] Taneja, I.J., "On Characterization of Generalized Information Measures" JCISS Vol.9 (3) 169-174, (1984).

[11] Taneja, I.J., "A joint characterization of directed divergence, inaccuracy and their generalization. J. Statist. Phys. 11 (2), 169-172, (1975).

[12] Taneja, I.J., "On an axiomatic characterization of information improvement and its generalization". J.CISS : 1 (12) 69-79 (1976).

[13] Singh, R.P. and Bhardwaj, J.D., "On weighted Information Improve ment" (Communicated).

[14] Singh, R.P. and Bhardwaj, J.D., "On qualitative measure of variation of information of type $\beta$ " (Communicated).

[15] - "On parametric Weighted information Improvement" (To appear in Information 
[16] Theil, H,-"Economics and Information Theory". North Holland and Amsterdam. (1967).

[17] Singh, R.P. and Bhardwaj J.D., "Characterization of weighted Information Improvement due to 2 and finite subsequent revisions". (To appear in Information Sciences).

R.P. Singh

Mathematics Department, L.R. College, Sahibabad, (Ghaziabad) - 201005, INDIA.

J.D. Bhardwaj

Directorate of Education, Delhi Administration, INDIA. 